\title{
Color changes in beef meat during pan cooking: kinetics, modeling and application to predict turn over time
}

\author{
Jara Moya $^{1}\left(\right.$ D Silvia Lorente-Bailo ${ }^{2}$ Ana Ferrer-Mairal ${ }^{2} \cdot$ Miguel A. Martínez $^{1,3} \cdot$ Begoña Calvo $^{1,3} \cdot$ Jorge Grasa $^{1,3}$. \\ María L. Salvador ${ }^{2}$
}

Received: 5 May 2021 / Revised: 16 July 2021 / Accepted: 18 July 2021 / Published online: 7 August 2021

(c) The Author(s) 2021

\begin{abstract}
The kinetics of heat-induced color changes in beef meat was determined and implemented in a numerical model for doublesided pan cooking of steak. The CIELab color space was used to obtain the lightness (coordinate $L^{*}$ ) and the reddish tone (coordinate $a^{*}$ ) of the cooked meat. $L^{*}$ was the CIELab coordinate that contributed the most to the change in the absolute color. Two response surfaces were found to describe the evolution with time and temperature of both color coordinates, $L^{*}$ and $a^{*}$. The model results were successfully verified with experimental data of the two coordinates along the thickness of the meat for three degrees of cooking. The Root-Mean-Squared Errors (RMSE) for coordinate $L^{*}$ were 5.17 (very rare), 2.02 (medium rare) and 3.83 (done), and for coordinate $a^{*} 1.44$ (very rare), 1.26 (medium rare) and 0.89 (done). The applicability of the model for practical cooking purposes was illustrated by determining the optimum turn over time to achieve a similar color profile on both sides of the meat. The turn over time depended on the desired degrees of cooking, and were comprised between one-half and two-thirds of the final cooking time, increasing from very rare cooking degree to done cooking degree.
\end{abstract}

Keywords Color $\cdot$ Cooking $\cdot$ Beef meat $\cdot$ Finite elements $\cdot$ Food model $\cdot$ Kinetic model

\section{Introduction}

The color of beef as a parameter to establish the final time of cooking is highly questioned, since color depends on many factors. For example, high $\mathrm{pH}$, modified atmosphere packaging, rapid thawing and low fat content can prolong the characteristic redness of raw meat; in contrast, brownish hues may appear prematurely in meat which has been frozen in bulk, thawed for a long time or packed in oxygenrich packaging $[1,2]$. To guarantee microbiological safety, the cooking times for meat should be based on temperature. The United States Department of Agriculture, USDA,

Jara Moya

jaram@unizar.es

1 Aragón Institute of Engineering Research (i3A), Universidad de Zaragoza, Zaragoza, Spain

2 Plant Foods Research Group, Instituto Agroalimentario de Aragon IA2, Universidad de Zaragoza-CITA, Miguel Servet 177, 50013 Zaragoza, Spain

3 Centro de Investigación Biomédica en Red en Bioingeniería, Biomateriales y Nanomedicina (CIBER-BBN), Zaragoza, Spain recommends a temperature of at least $62.8^{\circ} \mathrm{C}$ for steaks and a rest time of three minutes before eating. However, preferences from the point of view of consumer sensory perception may not coincide with safe temperatures [3]. In a domestic environment, meat color is still used to define the degree of doneness. Thermometers are usually employed when the meat is baked, but not when cooking by contact with a hot surface $[4,5]$. In this type of cooking, it is not easy to accurately measure the temperature in the center of the meat. A deviation of $\pm 1 \mathrm{~mm}$ in the temperature sensor position can cause temperature changes of $3^{\circ}-5^{\circ} \mathrm{C}[6,7]$. For practical domestic cooking purposes, it would be very interesting to know the evolution of color inside the meat. This information, as a complement to the temperature data, would allow a better approximation to the degree of doneness desired by the consumer. The relationship between cooking time, temperature and color of the meat depends on many factors such as the $\mathrm{pH}[8,9]$, the source of the meat [10], the water holding capacity [11], the packaging conditions [2] or the cooking method [12]. Those factors influencing the internal color of cooked meats have been recently reviewed by [13]. Despite the difficulties posed by these factors, color changes caused by heating can be quantified by chemical reaction 
kinetics in the same way as for other quality attributes [14, 15] or meat properties such as water content [6]. To the best of our knowledge, the kinetics of color change by the heating of beef meat has not been clearly defined. There are some studies such as that of [16] in which the variation of the CIELab coordinate $a^{*}$ is described, but this does not include changes in lightness; or that of [17] in which no data below $65^{\circ} \mathrm{C}$ are shown. The kinetic equations linked to a computational model would allow simulation of the evolution of meat color during cooking and the application of the model to help with practical cooking. This is a challenge, since pan cooking modeling is complex mainly due to the difficulty of quantifying the heat transfer between the hot surface and the meat, and the need to turn the meat over. These key aspects have not been contemplated in the above-mentioned studies, since they correspond to oven cooking.

The main objectives of this work were: (i) to determine the kinetics of thermal color changes in beef meat, (ii) to define and validate a coupled heat and mass transfer model capable of describing the evolution of beef color during domestic pan cooking which includes the turn over process, and (iii) to predict the turning over time for the meat from model simulations to achieve a similar color profile throughout the thickness of the meat. To make the results as useful as possible, the kinetic study was carried out with beef under three different conservation conditions (fresh, refrigerated and frozen), and the model was experimentally verified for three degrees of doneness.

\section{Materials and methods}

\section{Materials and properties}

Three Longissimus dorsi muscles from 1-year-old Asturiana de los Valles heifers were acquired in a local market 7 days postmortem. Nine $17 \mathrm{~mm}$-thick steaks perpendicular to the longitudinal axis were cut from the middle part of each loin for pan cooking. The resulting 27 steaks were individually placed in zip lock bags and distributed in three homogeneous batches. One batch was frozen and stored for 7 days at $-20{ }^{\circ} \mathrm{C}$ (frozen) and another was kept refrigerated at $4{ }^{\circ} \mathrm{C}$ for 7 days (refrigerated) using in both cases a KGN36VW35 Combi Bosch household refrigerator (BSH, Munich, Germany). The third batch was cooked on the day of acquisition (fresh).

For color kinetics determination, 80 steaks with a thickness of $4 \mathrm{~mm}$ were cut from different longitudinal positions of the middle part of each loin. These fillets, in turn, were cut into pieces of approximately $8 \mathrm{~g}$ to ensure uniform and fast heating. In addition, for each conservation condition, samples were taken from the $17 \mathrm{~mm}$-thick steaks for moisture determination and $\mathrm{pH}$ measurement.

\section{Moisture content}

The moisture content of the meat was determined by measuring the weight loss of $5 \mathrm{~g}$ samples after drying in a convection oven at $105{ }^{\circ} \mathrm{C}$ for $16-24 \mathrm{~h}$, using the Association of Official Analytical Chemists (AOAC) method 950.46. Five samples were analyzed for each conservation condition and the results were expressed as $\mathrm{g}$ of water per $100 \mathrm{~g}$ of sample.

\section{$\mathrm{pH}$}

A Basic 20+ Crison pH meter (Hach, Loveland, EE.UU.) equipped with a penetrating electrode was used to measure, just before cooking, the $\mathrm{pH}$ of the meat in each one of five replicates for each conservation condition.

\section{Color}

For the determination of the color kinetics, the pieces of meat were placed in plastic bags and immersed in a thermostatic water bath (Digiterm S-150, JP Selecta, Abrera, Spain). They were kept for times from $10 \mathrm{~s}$ to $30 \mathrm{~min}$ at a given temperature from 30 to $130^{\circ} \mathrm{C}$ before being cooled in iced water for $10 \mathrm{~min}$. In the $100^{\circ}-130^{\circ} \mathrm{C}$ range, sunflower oil was used as a heating fluid to avoid the limitations caused by the evaporation temperature of the water $\left(100{ }^{\circ} \mathrm{C}\right)$. The color was determined by digital image analysis. The images were acquired with a Canon Scan Lide 210 (Canon, Tokyo, Japan) at 600 dpi resolution, and stored in JPEG format using the Canon MP Navigator EX 4.0 software. They were then processed with Matrox Inspector 8.0 software (Matrox Electronic Systems Ltd, Dorval, Canada). Calibration of the digital system was performed using the UNE 48-103-94 Spanish color norm. The correspondence between CIELab coordinates $\left(L^{*}, a^{*}\right.$ and $\left.b^{*}\right)$ and RGB values were calculated using a quadratic model [18]. Five replicates were analyzed for each time, temperature and storage condition.

\section{Cooking procedure}

Frozen steaks were thawed at $4{ }^{\circ} \mathrm{C}$ for $24 \mathrm{~h}$ inside the refrigerator and all the samples were tempered to $20{ }^{\circ} \mathrm{C}$ before cooking. Each steak was cut to obtain three pieces of approximately $43.7 \pm 6.7 \mathrm{~g}, 81 \pm 21 \mathrm{~mm}$ long, $26 \pm 1 \mathrm{~mm}$ wide and $17 \pm 2 \mathrm{~mm}$ high, see Fig. 1a. The pieces were individually cooked in a forged aluminum pan with a Teflon platinum non-stick coating of $210 \mathrm{~mm}$ in diameter (WMF, WMF Group GmbH, Geislingen an der Steige, Germany) placed on an induction hob using the frying sensor at level 5 (BOSCH Schott Ceran PXY675DW4E/01 model, BSH, Munich, Germany). The meat was placed in the pan when the images taken with an infrared thermal camera (875-2 model, Testo, Lenzkirch, Germany) indicated that the surface of the pan 
Fig. 1 a Samples of beef steak for the pan cooking procedure, $\mathbf{b}$ experimental setup for temperature and weight loss measurement during the cooking process and $\mathbf{c}$ final moment of cooking for a done degree of doneness

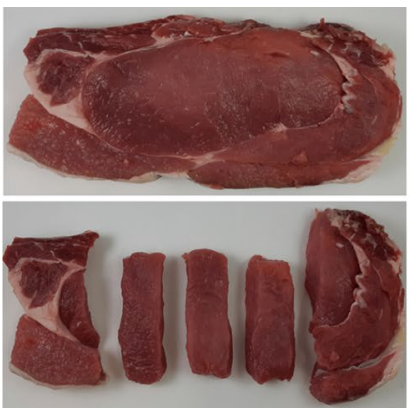

(a)

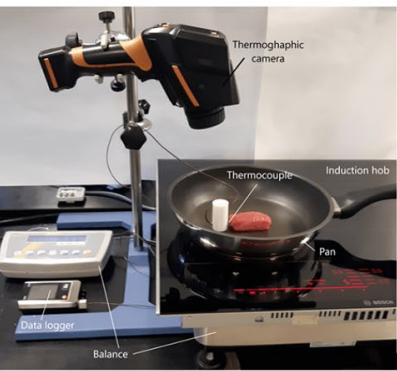

(b)

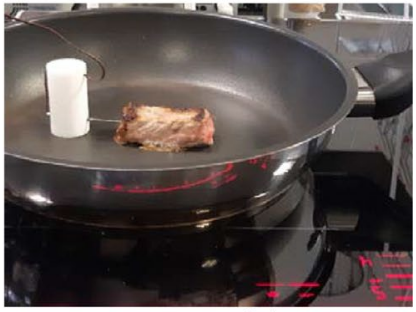

(c) had reached a temperature of $215 \pm 3{ }^{\circ} \mathrm{C}$ (Fig. 1b). The meat was cooked to three degrees of doneness: very rare, medium rare and done, which corresponded to cooking times of 180 s, $300 \mathrm{~s}$ and $420 \mathrm{~s}$, respectively. The samples were turned over at two-thirds of the total cooking time. Nine pieces were cooked for each degree of doneness and storage condition. Four of them were used to measure the evolution of the temperature in the center of the pieces by a penetration $\mathrm{T}$ type, $1.5 \mathrm{~mm}$-diameter thermocouple connected to a data logger (177-T4, Testo, Lenzkirch, Germany) and five to measure the meat weight loss during cooking using a balance (DS30K0.1L, Kern \& Sohn, Balinger-Frommern, Germany) on which the induction hob was placed. Two minutes after cooking, the pieces used to measure the weight loss were longitudinally cut, and then scanned following the same procedure as described in Section "Materials and properties". CIELab values were obtained at 21 different points along the thickness of the central region of the sample.

\section{Numerical simulation using a finite-element method}

The model developed with the software COMSOL Multiphysics 5.2a in [7] has been used to predict the color evolution in beef meat caused by pan cooking. This model (Fig. 2) reproduces the experimental tests and only a quarter of the pan and the meat due to the symmetry was considered. It is capable of reproducing the moment of turning over the steak by activating and deactivating the boundary conditions on the top and bottom surfaces related to the pan contact. The dimensions of the

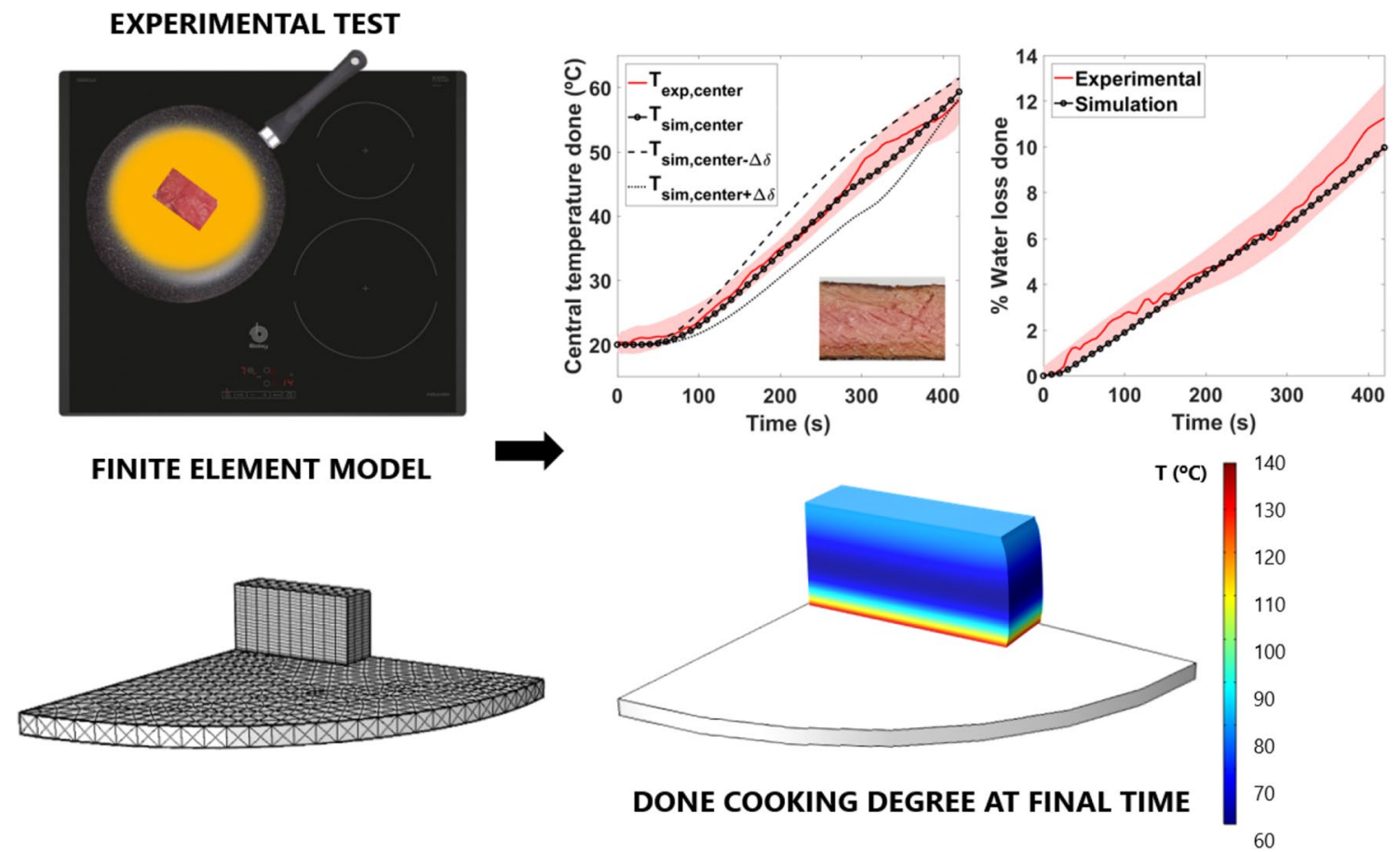

Fig. 2 Finite-element model and results obtained for temperature and water loss for a done degree of doneness 
piece of meat and the pan were set as in the experimental tests, as well as the three cooking times for the different degrees of doneness.

To reproduce the cooking process (Fig. 1c) and to obtain numerically the color acquired by the meat during cooking, a mathematical model which describes the physics underlying the main process is required.

The mathematical model reported in [7], in which the pan cooking of beef was described as a flow and transport problem in a deforming solid matrix during thermal processing, has been used in this study. The same considerations have been made, including the three physical phenomena which govern the problem: solid mechanics, heat transfer and mass transfer. The meat was considered as a continuum biphasic liquid-solid porous material, with the two phases having the same temperature at a given location, in which the solid matrix remained saturated. The moisture flows due to the swelling pressure caused by the shrinkage of the meat fibers and connective tissue.

In case of solid mechanics, the behavior of the meat during the cooking process has been approximated using the isotropic Neo-Hookean material model, in which the strain energy density function $\left(\Psi_{\mathrm{s}}\left(C_{\mathrm{s}}\right)\right)$ is defined as

$\Psi_{\mathrm{s}}\left(\boldsymbol{C}_{s}\right)=\Psi_{\mathrm{s}}\left(J_{\mathrm{s}}, \bar{C}_{\mathrm{s}}\right)=\frac{K}{2}\left(J_{\mathrm{s}}-1\right)^{2}+\frac{G^{\prime}}{2}\left(\bar{I}_{1}-3\right)$,

where $K$ and $G^{\prime}$ are the bulk and the shear elastic modulus, $J_{s}$ is the Jacobian of the motion and $\bar{I}_{1}=\operatorname{tr} \overline{\boldsymbol{C}}_{\mathrm{s}}$ is the first invariant of the modified (deviatoric) right Cauchy-Green tensor $\left(\overline{\boldsymbol{C}}_{\mathrm{s}}\right)$.

The heat transfer process inside the meat has been modeled using the energy balance equation for the entire product, as in [7]

$\left(\rho C_{\mathrm{p}}\right) \frac{\partial T}{\partial t}+\left(\boldsymbol{n}_{\mathrm{f}} \cdot \nabla\left(C_{\mathrm{p}, \mathrm{w}} T\right)\right)=\nabla \cdot\left(k_{\mathrm{p}} \nabla T\right)$,

where $\rho$ is the product density, and $C_{\mathrm{p}}$ and $C_{\mathrm{p}, \mathrm{w}}$ are the specific heat of the product and the water, respectively, whereas $k_{\mathrm{p}}$ is the thermal conductivity of the product, and $\boldsymbol{n}_{\mathrm{f}}$ is the water mass flux.

The mass conservation equation is

$$
\begin{aligned}
& \frac{\partial \phi_{\mathrm{f}} \bar{\rho}_{\mathrm{f}}}{\partial t}+\nabla \cdot\left(\phi_{\mathrm{f}} \bar{\rho}_{\mathrm{f}} \boldsymbol{v}_{\mathrm{s}}\right)=\nabla \cdot\left(D_{\mathrm{f}} \nabla\left(\phi_{f} \bar{\rho}_{\mathrm{f}}\right)\right. \\
& \left.\quad+D_{\mathrm{f}, \mathrm{T}} \nabla T\right),
\end{aligned}
$$

where $\phi_{\mathrm{f}}$ is the volume fraction for the liquid phase, $\bar{\rho}_{\mathrm{f}}$ is the intrinsic density of the fluid phase, $\boldsymbol{v}_{\mathrm{s}}$ corresponds to the absolute velocity of the solid phase, $D_{\mathrm{f}}=\rho_{\mathrm{f}} \frac{\kappa}{\mu} \vartheta$ is the diffusivity due to the water gradient concentration and $D_{\mathrm{f}, \mathrm{T}}=\rho_{\mathrm{f}} \frac{\kappa}{\mu} \vartheta \frac{\partial \rho_{\mathrm{f}, \mathrm{eq}}}{\partial T}$ is the diffusivity due to the temperature gradient, $\rho_{\mathrm{f}}$ being the density of the fluid phase, $\kappa$ the permeability of the medium, $\mu$ its dynamic viscosity, and $\vartheta$ a constant of proportionality.

The heat flow between the pan and the meat has been simulated, taking into account the boundary conditions. The contact equation and the heat transfer general equation are defined as

$$
\begin{aligned}
& -\left.k_{\mathrm{pan}} \frac{\partial T}{\partial z}\right|_{z_{\mathrm{pan}}=0}=-\left.k_{\mathrm{p}} \frac{\partial T}{\partial z}\right|_{z_{\text {meat }}=0} \\
& =H_{\mathrm{c}}\left(T_{\mathrm{pan}}-T_{\text {surf }}\right) \\
& q_{\text {surf }}=h\left(T_{\mathrm{amb}}-T_{\text {surf }}\right)-\lambda n_{\mathrm{f}, \text { surf }} \\
& -\mathbf{n}_{\mathrm{f}} C_{\mathrm{p}, \mathrm{w}} T \cdot \mathbf{N}_{\text {surf }},
\end{aligned}
$$

where $T_{\text {pan }}$ and $T_{\text {surf }}$ are the temperature of the pan and the temperature of the meat on the surface where the boundary condition is being evaluated, respectively. $k_{\text {pan }}$ is the conductivity of the pan and $\mathbf{N}_{\text {surf }}$ is the normal vector to the surface. $H_{c}$ is the thermal conductance between both surfaces and $h$ is the thermal convection coefficient. $T_{\mathrm{amb}}$ is the temperature of the air surrounding the meat, $\lambda$ is the latent heat of vaporization and $n_{\mathrm{f} \text {,surf }}$ the magnitude of the evaporation flux. On the surface in contact with the pan, the evaporation of water and the dripping have been considered (Eq. 5). On the upper face of the meat, neither of these phenomena occur (only the first term of Eq. 5 is applied), while on the side wall of the meat, only dripping has been taken into consideration (first and third therms of Eq. 5 are considered). The parameters and variables used in the model retain the same values as in [7]. The model was able to reproduce the evolution of the temperature in the central point of the meat and the loss of water registered in the experimental tests for different degrees of cooking.

\section{Results and discussion}

\section{Experimental determination of color kinetics}

The color of the beef changes during heating from red to light brown passing through pink and grayish, and these variations are reflected in the new scanned CIELab coordinates. Figure 3 shows, for fresh samples, the evolution of the coordinates $L^{*}, a^{*}$, and $b^{*}$ with the heating time at different temperatures expressed as normalized parameters relative to their initial values $\left(L_{\mathrm{o}}^{*}=45.23 \pm 0.99, a_{\mathrm{o}}^{*}=13.55 \pm 0.63\right.$ and $\left.b_{\mathrm{o}}^{*}=12.92 \pm 0.71\right)$. The lightness, $L^{*}$, at temperatures below $60{ }^{\circ} \mathrm{C}$, increases with time until it stabilizes, while at higher temperatures, the lightness rises quickly to a maximum and subsequently decreases until it levels off at a value lower than this maximum. At temperatures above $100{ }^{\circ} \mathrm{C}$, the lightness stabilizes at lower values, even lower than the initial 


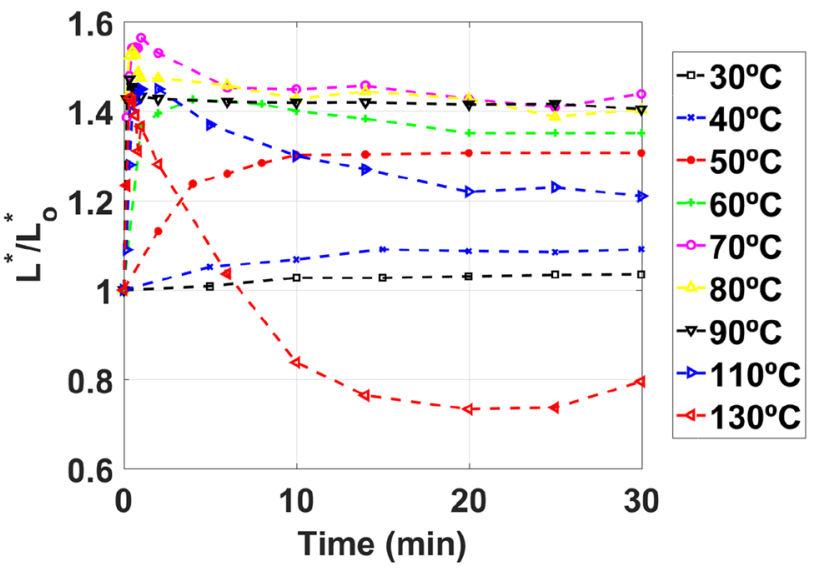

(a)

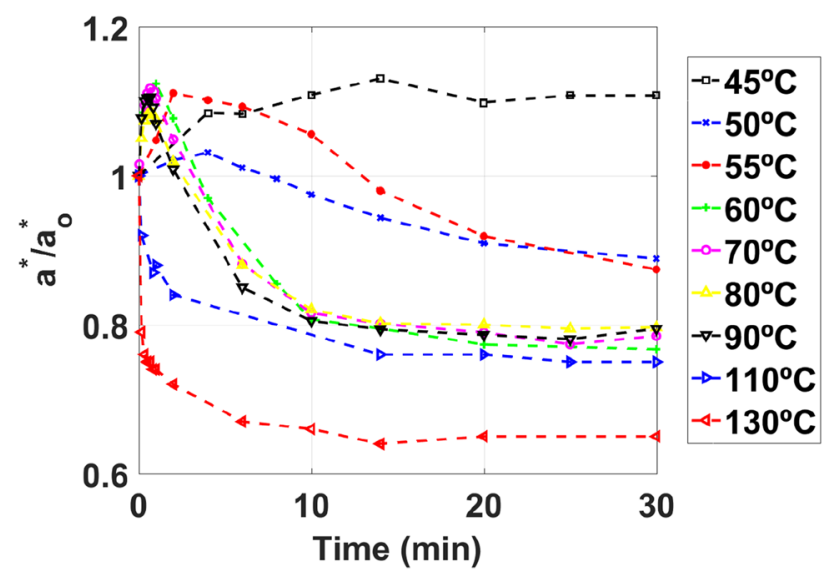

(b)

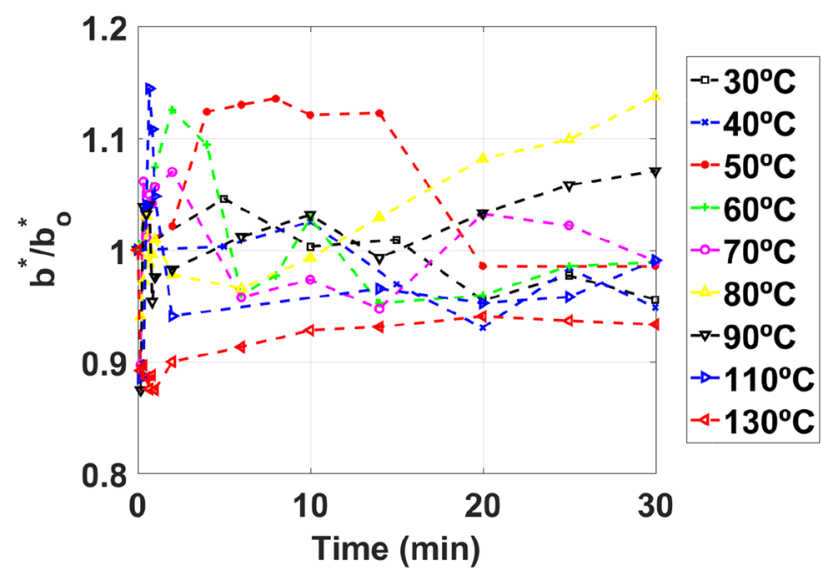

(c)

Fig. 3 Experimental values for a $L^{*} / \mathrm{L}_{\mathrm{o}}^{*}, \mathbf{b} a^{*} / \mathrm{a}_{\mathrm{o}}^{*}$ and $\mathbf{c} b^{*} / \mathrm{b}_{\mathrm{o}}^{*}$ over time and for different temperatures

one. Regarding the coordinate $a^{*}$, no changes were observed for temperatures below $45^{\circ} \mathrm{C}$. At higher temperatures, the values of $a^{*}$ follow a trend similar to coordinate $L^{*}$ (lightness); after a slight increase in the initial instants, a decrease toward an equilibrium value (always lower than the initial one) is observed due to the loss of the characteristic red hue of the raw meat. Above $100{ }^{\circ} \mathrm{C}$, the coordinate $a^{*}$ does not reach a maximum, but decreases with time until reaching an equilibrium value that is lower as the temperature increases. The $b^{*}$ coordinate during the warm-up time shows minor changes from the initial values without a clear trend.

The redness of the meat is associated with the heme proteins, and the loss during heating of the characteristic redness of raw meat is related to the denaturation of myoglobin and hemoglobin that occurs at $65^{\circ}-80{ }^{\circ} \mathrm{C}$ [19]. However, the change in the color of the meat during its heating cannot be explained exclusively by this. The greatest contribution to absolute color change is provided by lightness rather than by redness [20], as can be seen from the data in Fig. 3, especially at temperatures below $60{ }^{\circ} \mathrm{C}$. The increase in luminosity can be explained by the structural changes that meat undergoes during heating which modify the scattering of the light. The gaps generated between the fibers due to the muscle and myofibril shrinkage caused by protein denaturation, reduction of the water holding capacity and disruption of the sarcolemma, are some key structural changes that increase the reflected light [11]. The lightness changes which occur during heating are similar to the results found by [17] for beef meat at 66,98 and $205^{\circ} \mathrm{C}$. However, the evolution of the redness differs from that obtained by [16] since the coordinate $a^{*}$ decreased over time for all temperatures to reach a plateau at long cooking times. This could be described with a single fractional conversion first-order kinetics. However, in the cited work, data are not available at times shorter than $2.5 \mathrm{~min}$, being at those times when the increase in redness occurs.

\section{Influence of the domestic storage conditions on meat color}

The moisture content of the raw samples was $74.61 \pm 0.58$ $\%, 74.10 \pm 0.65 \%$ and $72.29 \pm 0.77 \%$ for the fresh, refrigerated and frozen samples, respectively. This means that during the defrosting process, the frozen meat lost $3.1 \%$ of its water content compared to fresh meat, while the refrigeration hardly affected it.

The CIELab coordinates values of the raw samples just before heat treatment $\left(L_{0}^{*}, a_{0}^{*}, b_{0}^{*}\right)$ for fresh, refrigerated and frozen meat were, respectively: $45.23 \pm 0.99,46.43 \pm 0.50$ and $45.44 \pm 0.51$ for $L_{0}^{*}, 13.55 \pm 0.63,13.27 \pm 0.30$ and $13.11 \pm 0.28$ for $a_{0}^{*}$ and $12.92 \pm 0.71,13.14 \pm 0.25$ and $13.87 \pm 0.42$ for $b_{0}^{*}$. Refrigeration or freezing, in the evaluated conditions, hardly influenced the color of the raw meat. There were no significant differences in redness between the three conditions $(p>0.05)$ indicating that the rate of oxidation at which oxymyoglobin changed into metmyoglobin was slow during the storage time [21]. On the other hand, 
the percentage of denatured myoglobin in beef muscle when heated is $\mathrm{pH}$-dependent, decreasing at high $\mathrm{pH}$ [9]. In this case, there were no significant differences $(p>0.05)$ in $\mathrm{pH}$ between the conditions before heat treatment: $5.64 \pm 0.05$, $5.62 \pm 0.07,5.63 \pm 0.05$ for the fresh, refrigerated and frozen samples, respectively.

The absolute color evolution, $\Delta E^{*}$, was calculated for fresh, refrigerated and frozen meat from the data of raw $\left(L_{\mathrm{o}}^{*}, a_{\mathrm{o}}^{*}, b_{\mathrm{o}}^{*}\right)$ and thermally treated samples $\left(L^{*}, a^{*}, b^{*}\right)$ using the following expression for the three storage conditions:

$\Delta E^{*}=\sqrt{\left(L_{\mathrm{o}}^{*}-L^{*}\right)^{2}+\left(a_{\mathrm{o}}^{*}-a^{*}\right)^{2}+\left(b_{\mathrm{o}}^{*}-b^{*}\right)^{2}}$.

$\Delta E^{*}$ data at different times and temperatures are shown in Fig. 4. The absolute color follows the same trend as the lightness, indicating that this is the CIELab coordinate that changes the most during heating. The absolute color evolution for the refrigerated or frozen samples was similar to that of fresh meat. Differences in absolute color between the conditions were small, $\Delta E^{*}<1$ at practically all times and temperatures, indicating that they were not visually detectable [22]. Therefore, color kinetics obtained from fresh samples could be applied to predict color changes during cooking regardless of the domestic preservation method used, if the tested conditions are similar to those presented here.

\section{Kinetic modeling}

By similarity to a chemical reaction, the evolution of $L^{*}$ and $a^{*}$ with time and temperature could be described by two successive fractional conversion first-order reactions (see 5.1). However, the kinetic parameters thus obtained (see Tables 3 and 4 in the annex) did not show a clear trend with temperature (for example, the kinetic constants did not follow an Arrheniustype dependence) that would allow a continuous function to be obtained that could be easily implemented in the finite-element model. Consequently, another alternative was proposed to describe the evolution of $L^{*}$ and $a^{*}$. With the experimental results, a response surface of each of the coordinates against time and temperature has been fitted.

Figure 5 shows these response surfaces. Equations 7 and 8 have been used in the finite-element model to calculate the coordinates $L^{*}$ and $a^{*}$ as a function of the maximum temperature reached at each point of the meat and the time these points remain at that temperature.

The equation which represents the response surface of coordinate $L^{*}$ is a five-degree polynomial function for temperature $T\left({ }^{\circ} \mathrm{C}\right)$ and linear for time $t(\mathrm{~min})$

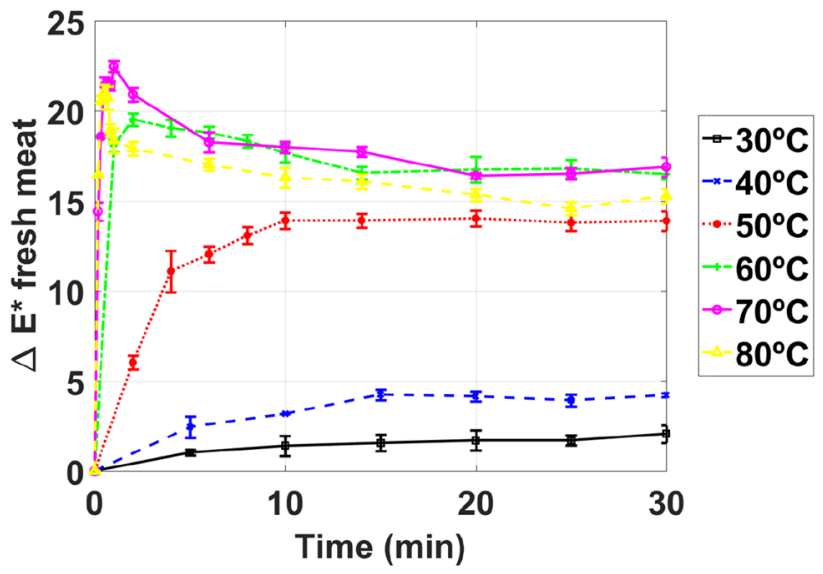

(a)

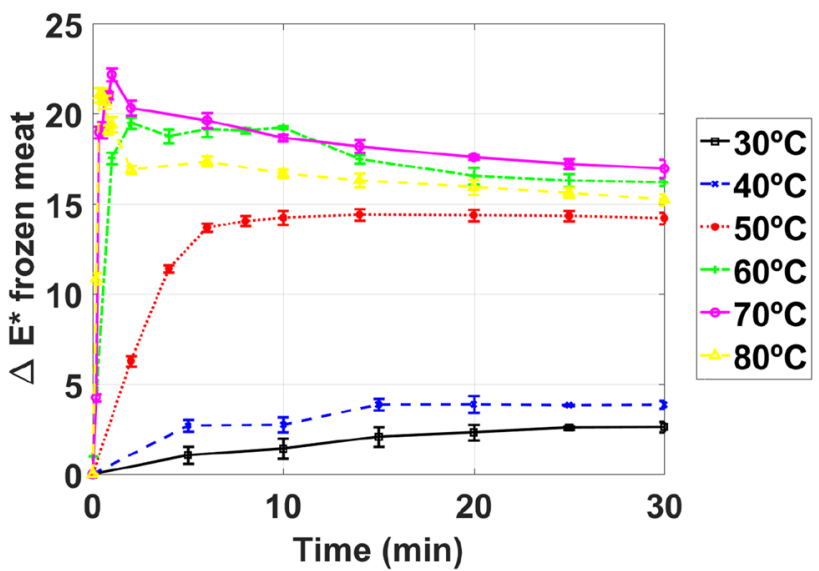

(b)

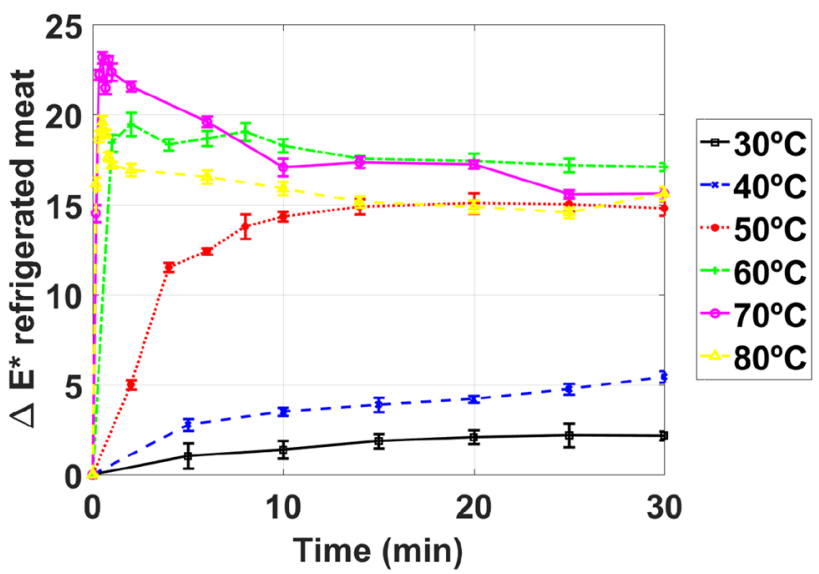

(c)

Fig. 4 Absolute color, $\Delta E^{*}$, evolution with heating time and temperature for $\mathbf{a}$ fresh, $\mathbf{b}$ refrigerated and $\mathbf{c}$ frozen beef 


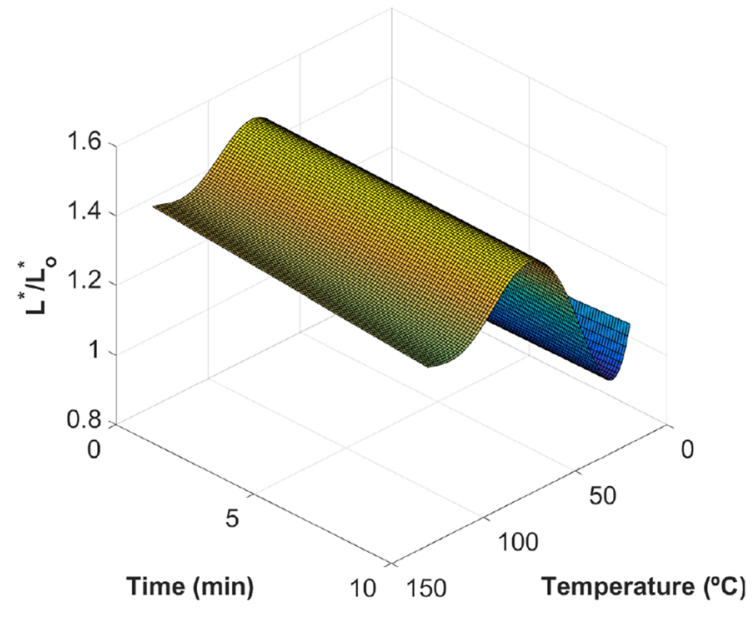

(a)

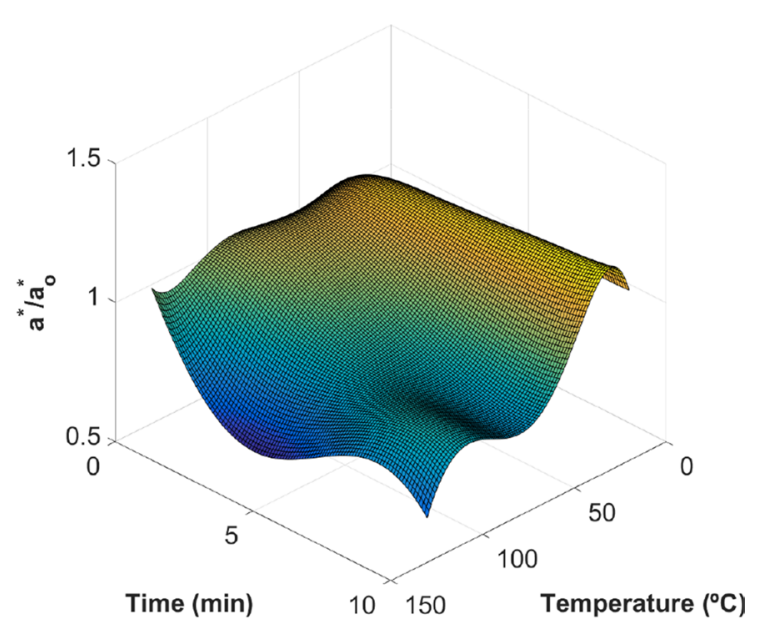

(b)

Fig. 5 a Response surface for $\frac{L^{*}}{L_{\mathrm{o}}^{*}}$. b) Response surface for $\frac{a^{*}}{a_{0}^{*}}$

Table 1 Coefficients for Eq. 7

\begin{tabular}{llllll}
\hline$L_{00}$ & 1.451 & $L_{10}$ & 0.06499 & $L_{01}$ & -0.01141 \\
$L_{20}$ & -0.315 & $L_{11}$ & -0.00843 & $L_{30}$ & 0.06247 \\
$L_{21}$ & -0.009398 & $L_{40}$ & 0.08047 & $L_{31}$ & -0.006208 \\
$L_{50}$ & -0.02618 & $L_{41}$ & 0.004121 & & \\
\hline
\end{tabular}

$$
\begin{array}{r}
\frac{L^{*}}{L_{\mathrm{o}}^{*}}=L_{00}+L_{10} \cdot T+L_{01} \cdot t+L_{20} \cdot T^{2} \\
+L_{11} \cdot T \cdot t+L_{30} \cdot T^{3}+L_{21} \cdot T^{2} \cdot t+L_{40} \cdot T^{4} \\
+L_{31} \cdot T^{3} \cdot t+L_{50} \cdot T^{5}+L_{41} \cdot T^{4} \cdot t .
\end{array}
$$

An $R$-square of 0.9661 corresponds to this adjustment. The coefficients of Eq. 7 are shown in Table 1:

Meanwhile, in the case of coordinate $a^{*}$, this surface is represented by a polynomial function of degree five for both temperature $T\left({ }^{\circ} \mathrm{C}\right)$ and time $t(\mathrm{~min})$

$$
\begin{aligned}
& \frac{a^{*}}{a_{\mathrm{o}}^{*}}=a_{00}+a_{10} \cdot T+a_{01} \cdot t+a_{20} \cdot T^{2}+a_{11} \cdot T \cdot t+a_{02} \cdot t^{2} \\
& +a_{30} \cdot T^{3}+a_{21} \cdot T^{2} \cdot t+a_{12} \cdot T \cdot t^{2}+a_{03} \cdot t^{3} \\
& +a_{40} \cdot T^{4}+a_{31} \cdot T^{3} \cdot t+a_{22} \cdot T^{2} \cdot t^{2}+a_{13} \cdot T \cdot t^{3} \\
& +a_{04} \cdot t^{4}+a_{50} \cdot T^{5}+a_{41} \cdot T^{4} \cdot t+a_{32} \cdot T^{3} \cdot t^{2} \\
& +a_{23} \cdot T^{2} \cdot t^{3}+a_{14} \cdot T \cdot t^{4}+a_{05} \cdot t^{5} .
\end{aligned}
$$

Regarding the goodness of the fit, a R-square of 0.9184 was obtained. The coefficients of Eq. 8 are shown in Table 2:
Table 2 Coefficients for Eq. 8

\begin{tabular}{llllll}
\hline$a_{00}$ & 0.9453 & $a_{10}$ & -0.1039 & $a_{01}$ & -0.1819 \\
$a_{20}$ & 0.06826 & $a_{11}$ & -0.001362 & $a_{02}$ & 0.03775 \\
$a_{30}$ & -0.05929 & $a_{21}$ & 0.1845 & $a_{12}$ & 0.07191 \\
$a_{03}$ & 0.03524 & $a_{40}$ & -0.03432 & $a_{31}$ & 0.01048 \\
$a_{22}$ & 0.03851 & $a_{13}$ & -0.04799 & $a_{04}$ & -0.05117 \\
$a_{50}$ & 0.01798 & $a_{41}$ & -0.03213 & $a_{32}$ & -0.00261 \\
$a_{23}$ & -0.04949 & $a_{14}$ & -0.005256 & $a_{05}$ & 0.02642 \\
\hline
\end{tabular}

\section{Validation of the computational model for color prediction}

The kinetic model was coupled to the heat and mass transfer model to simulate the change of color during the process. Experimental values of coordinates $L^{*}$ and $a^{*}$ along the thickness of the pieces of beef were collected with the purpose of comparing them with the numerical results. This comparison is shown in Figs. 6 and 7. The predictions are in good agreement with the experimental data: the RootMean-Squared Errors, RMSE, for coordinate $L^{*}$ are 5.17 (very rare), 2.02 (medium rare) and 3.83 (done), and for coordinate $a^{*}$ are 1.44 (very rare), 1.26 (medium rare) and 0.89 (done). In this manner, this validation allowed the model to be verified and the assumptions made to be considered appropriate.

\section{Evolution of color during cooking for different turn over times}

Figure 8 shows digital images of the different cooking degrees for the final time of cooking. For short cooking 
Fig. 6 Experimental and numerical results for coordinate $L^{*}$ at a very rare, $\mathbf{b}$ medium rare and c done degrees of cooking

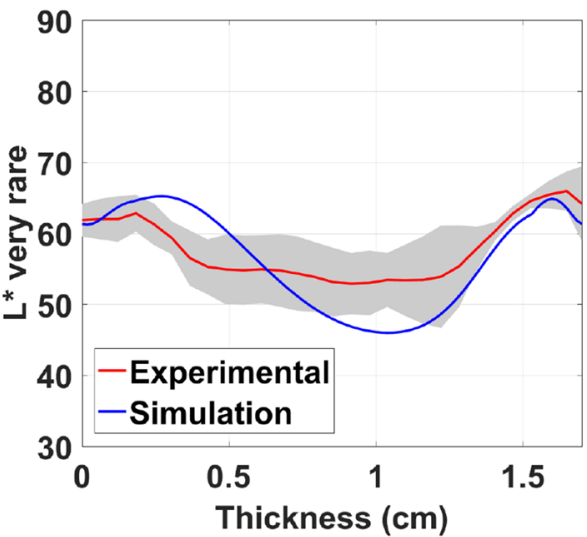

(a)

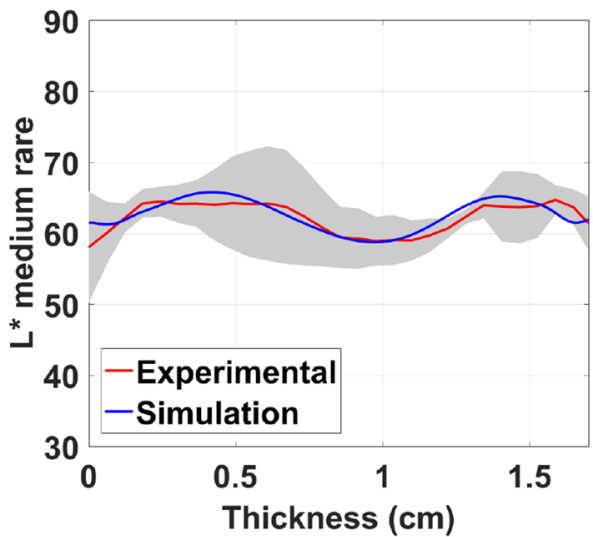

(b)

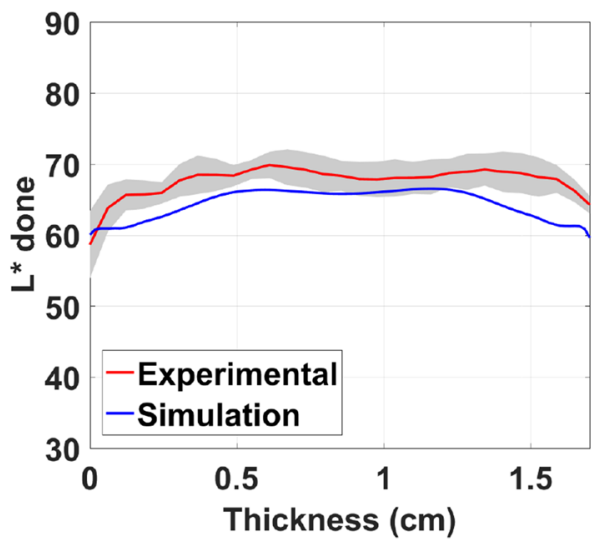

(c)

times, two zones can be visually distinguished in these images: a central one with slightly higher luminosity and redness than raw meat ( $L^{*}$ around 55 and $a^{*}$ between 14 and 15, see Figs. 7 and 6), and another narrow area close to the surface in which the meat has lost its characteristic redness ( $a^{*}$ lower than 13.5). For longer cooking times, the thickness of the area where the meat loses its characteristic red hue increases, acquiring more intense grayish-brownish tones (the $a^{*}$ coordinate in this area drops down to 8), while the luminosity of the central area reaches higher values $\left(L^{*}\right.$ between 65 and 70).

Once the model has been validated, it is possible to know the evolution of the meat color over time and determine the turn over time of the meat for which the degree of cooking on both sides of the meat would be similar; that is, to achieve a symmetrical distribution of the two areas that are visually appreciated throughout the thickness of the meat. To obtain more qualitative results, a conversion to RGB coordinates has been made from coordinates $L^{*}$ and $a^{*}$, considering the coordinate $b^{*}$ constant with a value equal to that of raw meat. These calculations were done using the open-source ColorMine software. In this way, the color of the meat has been recreated for different instants and turn over times (Fig. 9). Similar to what was observed visually in the photographs of the meat (Fig. 8), an area near the surface can be distinguished in which increasingly brownish tones appear over time and also a central area with increasing luminosity over time.

As a consequence of these color changes of the meat during the cooking process, the absolute color $\left(\Delta E^{*}\right)$ at the end of the cooking process in the central zone of the meat is at a minimum for the very rare and a maximum for the done degrees of cooking. In Fig. 10, the absolute color at the end of the cooking throughout the thickness of the meat is shown for different turning times and cooking degrees. For the very rare condition, the absolute color in the center of the meat has hardly changed with respect to the raw meat ( $\Delta E^{*}$ around 2$)$, while as we approach both extremes, the absolute color increases, reaching a maximum value, and then decreases sharply in the outermost area of the meat. For the medium rare and done degrees of cooking, the absolute color profile is similar, but the absolute color in the center of the meat is between 7 and 15 for medium rare and around 21 for done. In all cases, the points within the meat in which the absolute color begins to decrease depend on the turn over time. 
Fig. 7 Experimental and numerical results for coordinate $a^{*}$ at a very rare, $\mathbf{b}$ medium rare and $\mathbf{c}$ done degrees of cooking

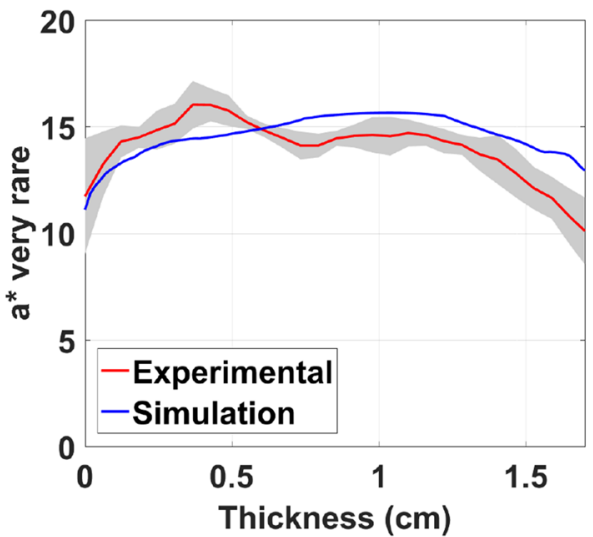

(a)

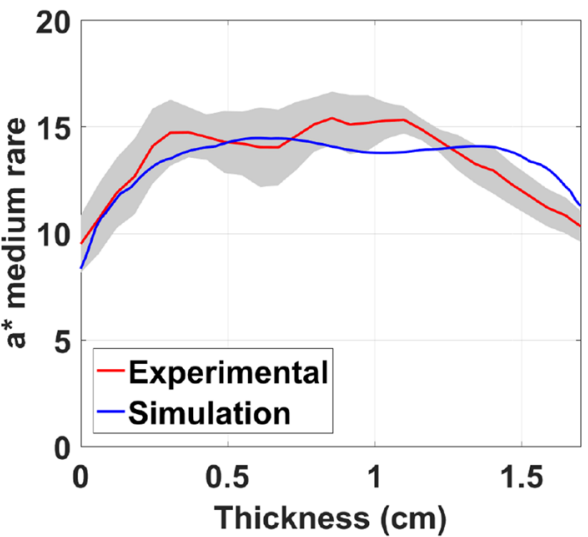

(b)

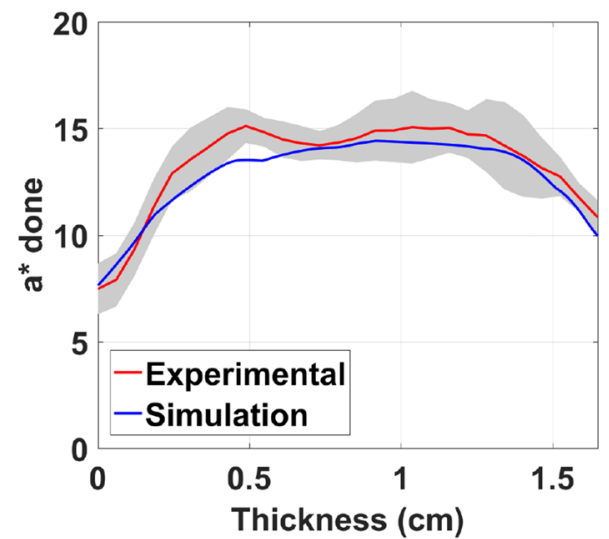

(c)

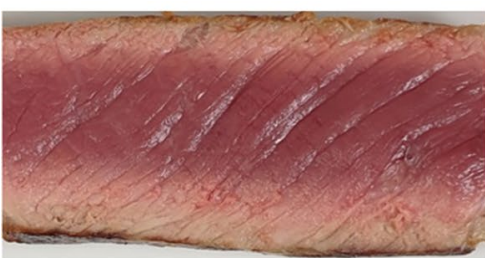

(a)

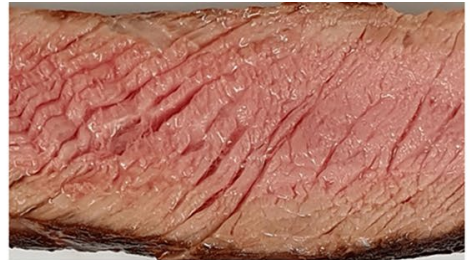

(b)

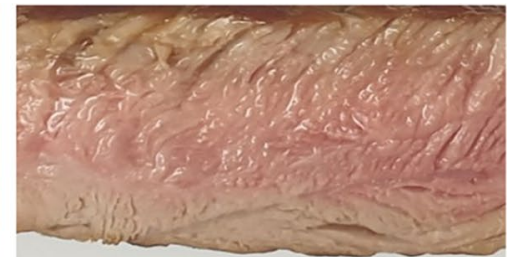

(c)

Fig. 8 Experimental color for the final time of cooking of $\mathbf{a}$ very rare, $\mathbf{b}$ medium rare and $\mathbf{c}$ done degrees of cooking. Turn over at two-thirds of the total cooking time

In Fig. 11, the final appearance of the color in the interior of the meat is recreated as described above for different degrees of cooking and turn over times. The positions in which the absolute color begins to decrease (obtained from the model results) are indicated with a blue line and the distance of those points to the edge of the meat is also indicated. As can be observed, for short cooking times (very rare), a product with a similar doneness on both sides is achieved when turn over occurs half way through the total cooking time. For the medium rare and done degrees of cooking, the symmetry in color is achieved when the turn over time is three-fifths and two-thirds of the total cooking time, respectively.

\section{Conclusions}

Changes in the color of beef during cooking are usually identified by the transition from reddish to brownish tones associated with the modifications suffered by heme proteins. However, in this study, luminosity was the color coordinate that contributed the most to the change in absolute color, 
Fig. 9 Evolution of the meat color along its thickness over time and depending on the turn over time
TURN OVER AT $1 / 2$

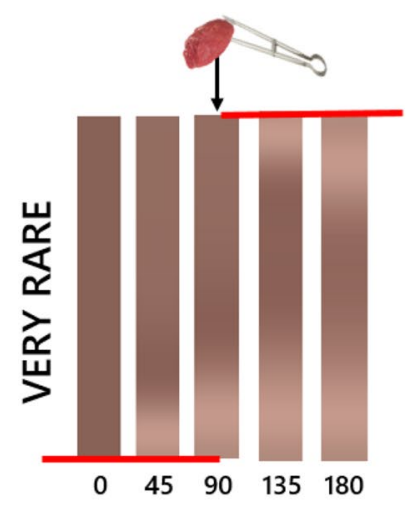

(s)

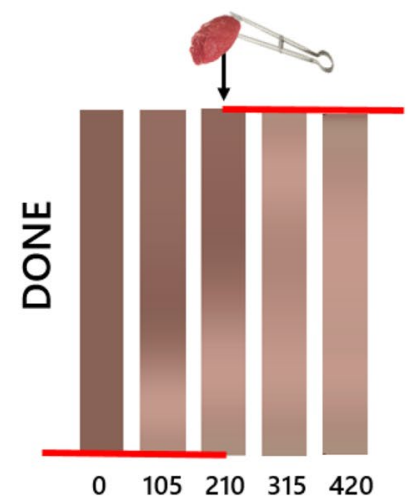

(s)

\section{TURN OVER AT 2/3}

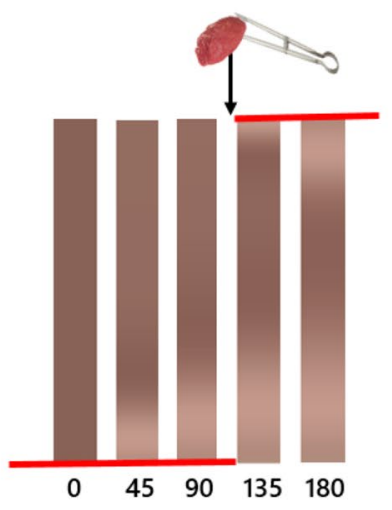

(s)

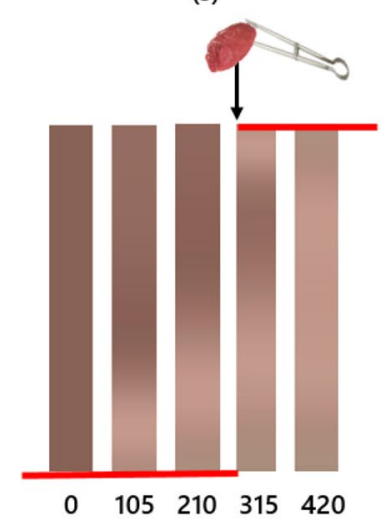

(s)
TURN OVER AT 4/5
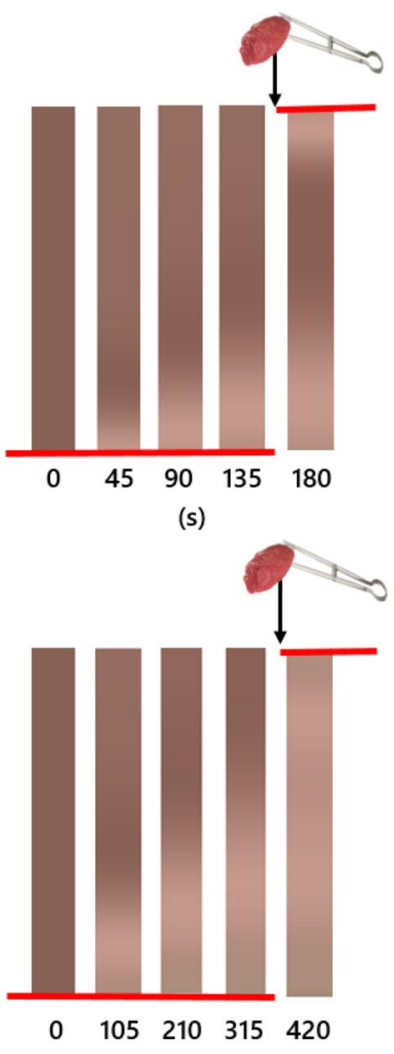

(s) confirming the importance of structural changes that modify the dispersion of light in the color of cooked meat. The evolution of the CIELab coordinates, $L^{*}$ and $a^{*}$, during beef cooking has been described by means of a response surface method which has allowed the implementation of beef color changes in a computational model [7]. The different conditions of conservation tested (fresh, refrigerated and frozen), typical in the domestic environment, did not influence the evolution of the absolute color of the meat during the heat treatment sufficiently to be visually appreciated, so that in these conditions, the kinetics obtained were able to be applied.

The proposed 3D mathematical model considers the heat and moisture transfer phenomena, simultaneously with the meat deformation, during the two-sided pan cooking of beef, and has the kinetics of color change linked to it. The model allowed us to adequately predict the color changes of the meat as shown by the good agreement between the numerical and experimental results for the three degrees of doneness tested. The profile of the luminosity along the thickness depends on the degree of doneness, being at a minimum in the center for short cooking times (very rare) and at a maximum for longer cooking times (medium rare and done). However, in all cases, there is a decrease in the value of the luminosity, and consequently of the absolute color, at points close to the surface. The location of these points depends on the time at which the meat has been turned over. The applicability of the model for practical cooking purposes has been illustrated by the calculation of the turn over time, so that the steaks are cooked evenly on both sides. Depending on the degree of doneness desired, the turn over time would change.

\section{Annex}

\section{A kinetic model}

The changes in food attributes by thermal treatments can be expressed by a kinetic model [23], Eq. 9 and 10

$\frac{\mathrm{d} Q}{\mathrm{~d} t}=-k\left(Q-Q_{\infty}\right)^{n}, \quad Q_{0} \geq Q \geq Q_{\infty}$

$\frac{\mathrm{d} Q}{\mathrm{~d} t}=k\left(Q_{\infty}-Q\right)^{n}, \quad Q_{0} \leq Q \leq Q_{\infty}$,

where $Q$ is the measured coordinate, $t$ is time, $n$ is the reaction order (mostly $n=0,1$ or 2), $Q_{\infty}$ is the final non zero equilibrium quality value and $k$ is the reaction rate constant, 
Fig. 10 Evolution of absolute color, $E^{*}$, along meat thickness over time and depending on the turn over time for a very rare, $\mathbf{b}$ medium rare and $\mathbf{c}$ done degrees of cooking

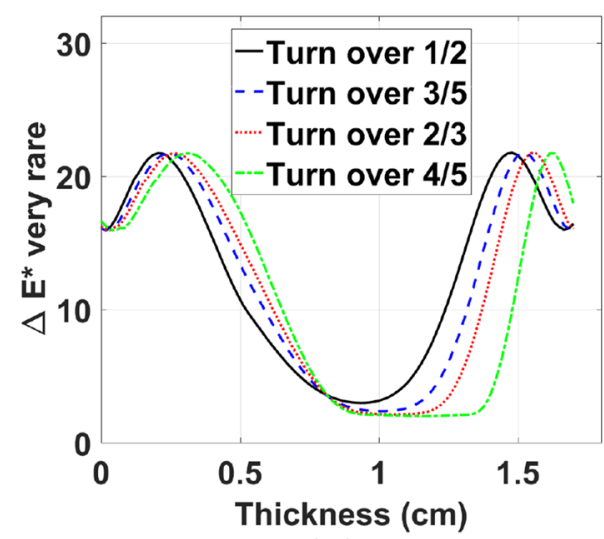

(a)

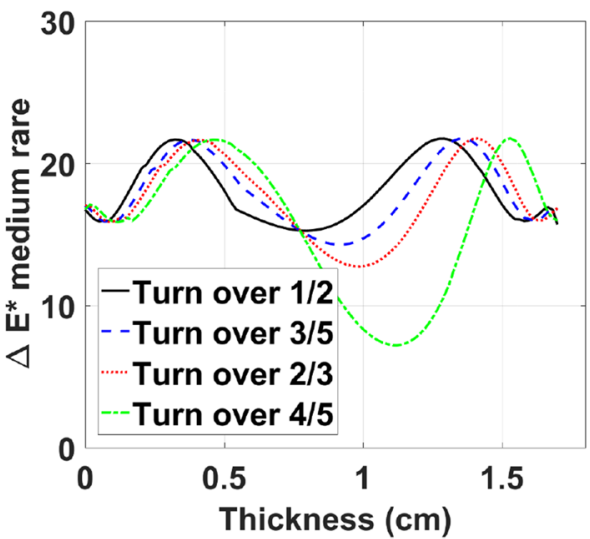

(b)

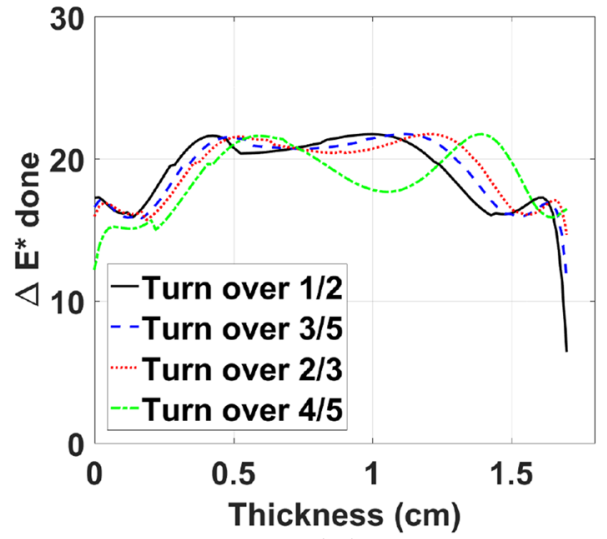

(c)

Fig. 11 Final color of the meat for each turn over time in case of a very rare, $\mathbf{b}$ medium rare and $\mathbf{c}$ done degree of cooking. Blue lines indicate the positions in which the absolute color begins to decrease. Lower side of the meat in the figure was put in contact with the pan first

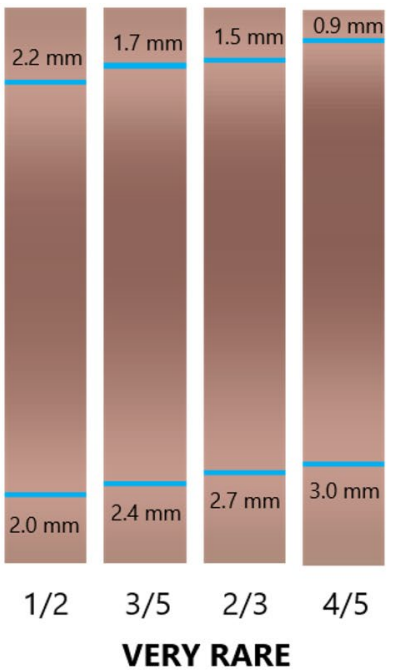

VERY RARE
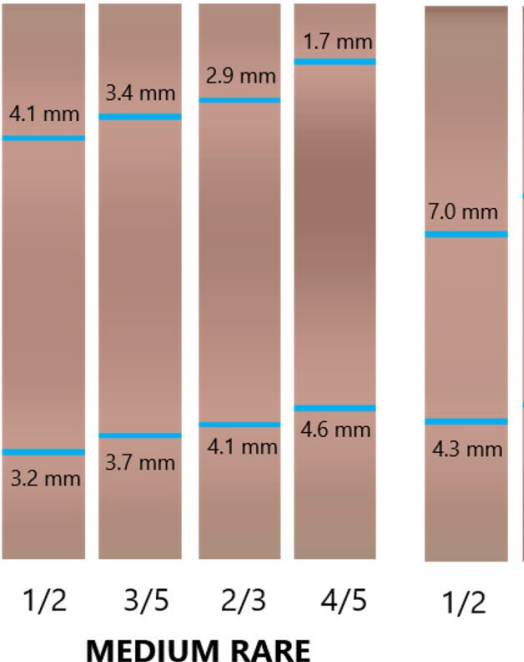

$1 / 2$

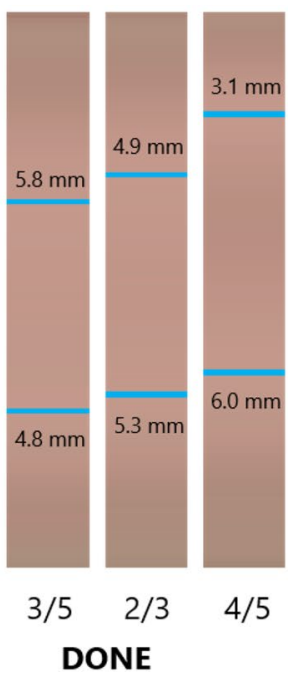

which is temperature dependent, usually expressed by the Arrhenius equation (Eq. 11):

$k=k_{0} \exp \left(\frac{-E_{\mathrm{a}}}{R T}\right)$, where $T\left({ }^{\circ} \mathrm{C}\right)$ is the absolute temperature, $k_{0}$ is the preexponential factor, $E_{\mathrm{a}}$ is the activation energy and $R$ is the universal gas constant $(8.31 \mathrm{~J} / \mathrm{molK})$.

However, the evolution with time of the $L^{*}$ and $a^{*}$ color coordinates during the thermal treatment of beef cannot be described by a single reaction taking into account that these 


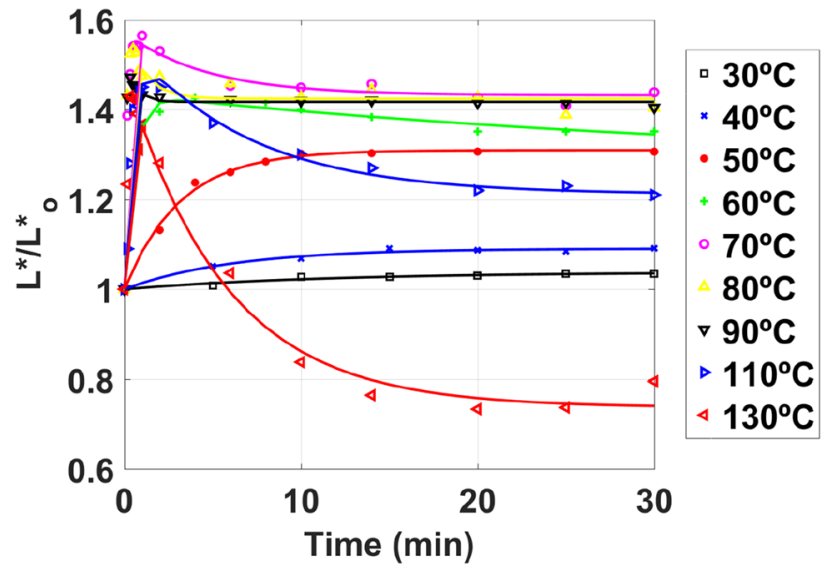

(a)

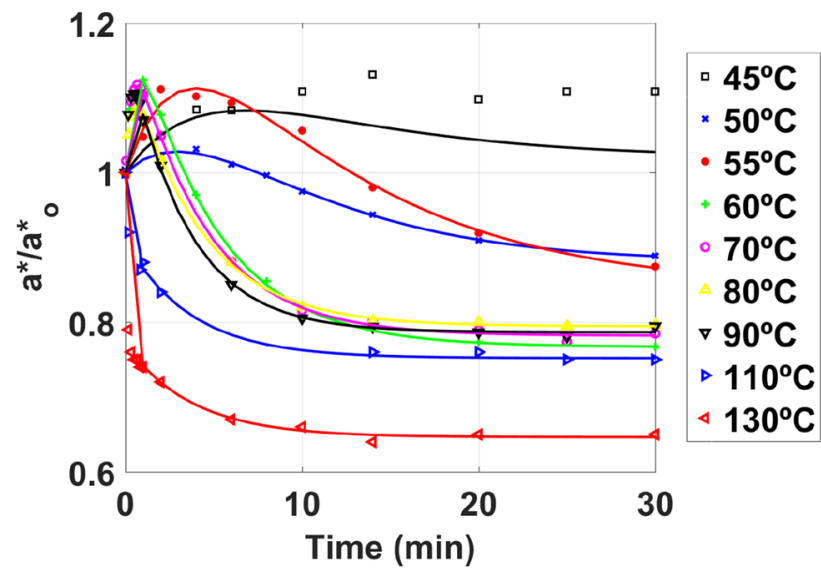

(b)

Fig. 12 Adjustment of the kinetic model for $\mathbf{a} L^{*} / L_{\mathrm{o}}^{*}$ and $\mathbf{b} a^{*} / a_{\mathrm{o}}^{*}$ for different temperatures

coordinates reach a maximum (Fig. 3). The changes in $L^{*}$ and $a^{*}$ during the cooking of beef were assumed to be those of the intermediate attribute, $Q$, involved in two successive reactions according to the following scheme:

$$
\begin{array}{llll} 
& R \stackrel{k_{1}}{\longrightarrow} Q \stackrel{k_{2}}{\longrightarrow} P \\
t=0 & R_{0} & Q_{0} & P_{0} \\
t=\infty & R_{\infty} & Q_{\infty} & P_{\infty}
\end{array}
$$

where $R_{\mathrm{o}}, Q_{\mathrm{o}}$ and $P_{\mathrm{o}}$ were the initial values of the attributes $R, Q$ and $P$, and $R_{\infty}, Q_{\infty}$ and $P_{\infty}$ were the final non-zero equilibrium quality values after long heating times.

Therefore, the evolution of $L^{*}$ and $a^{*}$ with time and temperature was described by a kinetics model based on two successive fractional conversion first-order reactions, Eqs. 12 and 13

$$
\begin{aligned}
\frac{L^{*}}{L_{0}^{*}} & =1+\left(\frac{L_{\infty}^{*}}{L_{0}^{*}}-1\right)\left(1-e^{-k_{2} t}\right) \\
& +\frac{k_{1}\left(R_{0}-R_{\infty}\right)}{L_{0}^{*}\left(k_{2}-k_{1}\right)}\left(e^{-k_{1} t}-e^{-k_{2} t}\right) \\
\frac{a^{*}}{a_{0}^{*}} & =1+\left(\frac{a_{\infty}^{*}}{a_{0}^{*}}-1\right)\left(1-e^{-k_{2} t}\right) \\
& +\frac{k_{1}\left(R_{0}-R_{\infty}\right)}{a_{0}^{*}\left(k_{2}-k_{1}\right)}\left(e^{-k_{1} t}-e^{-k_{2} t}\right),
\end{aligned}
$$

where $L_{\infty}$ and $a_{\infty}$ are the values of the coordinates at infinite time, $\mathrm{k}_{1}$ and $k_{2}\left(\mathrm{~min}^{-1}\right)$ are the rate constants, $\mathrm{t}(\mathrm{min})$ is the time, and $R_{0}-R_{\infty}$ is the reactant consumed in the first reaction. The kinetic parameters were obtained by minimization of the sum of squared differences between calculated and experimental values using the Levenberg-Marquardt algorithm. Tables 3 and 4 show the estimated kinetic parameters for both color CIELab coordinates. The color degradation rate constants increased with the temperature, but this dependence could not be accurately described by the Arrhenius equation. In all cases, the coefficient of determination $\left(\mathrm{R}^{2}\right)$ was greater than 0.9469 , indicating that the
Table 3 Kinetic parameters of thermal degradation of lightness $L^{*}$ for beef

\begin{tabular}{llllll}
\hline $\begin{array}{l}\text { Tempera- } \\
\text { ture }\left({ }^{\circ} \mathrm{C}\right)\end{array}$ & $n=\frac{L_{\infty}^{*}}{L_{0}^{*}}$ & $m=\frac{R_{0}-R_{\infty}}{L_{0}^{*}}$ & $k_{1}\left(\mathrm{~min}^{-1}\right)$ & $k_{2}\left(\mathrm{~min}^{-1}\right)$ & $R^{2}$ \\
\hline 30 & 1 & $0.0383 \pm 0.0044$ & $0.0014 \pm 0.0004$ & 0 & 0.9469 \\
40 & 1 & $0.0906 \pm 0.0032$ & $0.0027 \pm 0.0004$ & 0 & 0.9799 \\
45 & 1 & $0.1642 \pm 0.0033$ & $0.0031 \pm 0.0002$ & 0 & 0.9944 \\
50 & 1 & $0.3095 \pm 0.0057$ & $0.0053 \pm 0.0 .0004$ & 0 & 0.9934 \\
55 & 1 & $0.3339 \pm 0.0098$ & $0.0123 \pm 0.0017$ & 0 & 0.9731 \\
60 & $1.2684 \pm 0.2263$ & $0.4287 \pm 0.0112$ & $0.0305 \pm 0.0051$ & $0.0004 \pm 0.0009$ & 0.9887 \\
70 & $1.4320 \pm 0.0062$ & $0.5541 \pm 0.0086$ & $0.1100 \pm 0.0128$ & $0.0036 \pm 0.0011$ & 0.9656 \\
80 & $1.4244 \pm 0.0068$ & $0.5342 \pm 0.0179$ & $0.1527 \pm 0.0417$ & $0.0163 \pm 0.0082$ & 0.9687 \\
90 & $1.4172 \pm 0.0022$ & $0.4462 \pm 0.0063$ & $0.2009 \pm 0.0212$ & $0.0163 \pm 0.0080$ & 0.9977 \\
\hline
\end{tabular}


Table 4 Kinetic parameters of thermal degradation of redness $a^{*}$ for beef

\begin{tabular}{llllll}
\hline $\begin{array}{l}\text { Tempera- } \\
\text { ture }\left({ }^{\circ} \mathrm{C}\right)\end{array}$ & $n=\frac{a_{\infty}^{*}}{a_{0}^{*}}$ & $m=\frac{R_{0}-R_{\infty}}{a_{0}^{*}}$ & $k_{1}\left(\mathrm{~min}^{-1}\right)$ & $k_{2}\left(\mathrm{~min}^{-1}\right)$ & $R^{2}$ \\
\hline 45 & $1.0208 \pm 0.0270$ & $0.1885 \pm 0.0740$ & $0.0027 \pm 0.0031$ & $0.0027 \pm 0.0006$ & 0.98644 \\
50 & $0.8810 \pm 0.0093$ & $0.2467 \pm 0.0286$ & $0.0029 \pm 0.0003$ & $0.0029 \pm 0.0093$ & 0.9949 \\
55 & $0.8436 \pm 0.0411$ & $1.1803 \pm 0.1220$ & $0.0017 \pm 0.0010$ & $0.0050 \pm 0.0026$ & 0.9759 \\
60 & $0.7669 \pm 0.0058$ & $3.8473 \pm 0.6705$ & $0.0037 \pm 0.0 .0003$ & $0.0328 \pm 0.0043$ & 0.9970 \\
70 & $0.7820 \pm 0.0024$ & $4.3628 \pm 0.4253$ & $0.0041 \pm 0.0002$ & $0.0451 \pm 0.0035$ & 0.9992 \\
80 & $0.7945 \pm 0.0014$ & $3.9168 \pm 0.2684$ & $0.0042 \pm 0.0001$ & $0.0484 \pm 0.0026$ & 0.9996 \\
90 & $0.7866 \pm 0.0025$ & $5.4333 \pm 0.6253$ & $0.0050 \pm 0.0002$ & $0.0735 \pm 0.0066$ & 0.9989 \\
\hline
\end{tabular}

kinetic model proposed was suitable to describe the thermal degradation of the beef color.

Figure 12 shows the adjustment of the kinetic model described in this annexed with the experimental results described in Section "Experimental determination of color kinetics", for $a^{*} / a_{\mathrm{o}}^{*}$ and $L^{*} / L_{\mathrm{o}}^{*}$, and for different temperatures.

Funding This work has been funded by the Spanish Ministry of Science, Innovation and Universities through the RETOS-COLLABORATION 2017 program (project RTC-2017-5965-6, ARQUE), cofinanced by the European Union with ERDF; and by the BSH Home Appliances Group. It has also been supported by the Department of Industry and Innovation (Government of Aragon) through the research group Grant T24-20R and T07-20R (co-financed by Feder 2014-2020: Construyendo Europa desde Aragon). J. Moya was supported by the Government of Spain, order CNU/692/2019.

\section{Availability of data and materials Not applicable.}

\section{Declarations}

Conflicts of interest The authors declare that they have no competing interests.

Compliance with ethics requirements This article does not contain any studies with human or animal objects.

Code availability This study has been carried out using COMSOL Multiphysics 5.2a software.

Open Access This article is licensed under a Creative Commons Attribution 4.0 International License, which permits use, sharing, adaptation, distribution and reproduction in any medium or format, as long as you give appropriate credit to the original author(s) and the source, provide a link to the Creative Commons licence, and indicate if changes were made. The images or other third party material in this article are included in the article's Creative Commons licence, unless indicated otherwise in a credit line to the material. If material is not included in the article's Creative Commons licence and your intended use is not permitted by statutory regulation or exceeds the permitted use, you will need to obtain permission directly from the copyright holder. To view a copy of this licence, visit http://creativecommons.org/licenses/by/4.0/.

\section{References}

1. King N, Whyte R (2006) Does it look cooked? a review of factors that influence cooked meat color. J Food Sci 71:R31-R40

2. Stella S, Bernardi C, Tirloni E (2018) Influence of skin packaging on raw beef quality: a review. J Food Qual 1-9:2018

3. López Osornio MM, Hough G, Salvador A, Chambers E, McGraw S, Fiszman S (2008) Beef's optimum internal cooking temperature as seen by consumers from different countries using survival analysis statistics. Food Qual Prefer 19(1):12-20

4. Elshahat S, Woodside JV, McKinley MC (2019) Meat thermometer usage amongst European and North American consumers: a scoping review. Food Control 106:106692

5. McCurdy SM, Hillers V, Cann SE (2005) Consumer reaction and interest in using food thermometers when cooking small or thin meat items. Food Prot Trends 25:826-831

6. Kondjoyan A, Oillic S, Portanguen S, Gros JB (2013) Combined heat transfer and kinetic models to predict cooking loss during heat treatment of beef meat. Meat Sci 95(2):336-344

7. Moya J, Lorente-Bailo S, Salvador ML, Ferrer-Mairal A, Martínez MA, Calvo B, Grasa J (2021) Development and validation of a computational model for steak double-sided pan cooking. J Food Eng 298:110498

8. Hughes J, Clarke F, Purslow P, Warner R (2017) High pH in beef longissimus thoracis reduces muscle fibre transverse shrinkage and light scattering which contributes to the dark colour. Food Res Int 101:228-238

9. Trout GR (1989) Variation in myoglobin denaturation and color of cooked beef, pork, and turkey meat as influenced by $\mathrm{pH}$, sodium chloride, sodium tripolyphosphate, and cooking temperature. J Food Sci 54(3):536-540

10. Suman SP, Hunt MC, Nair MN, Rentfrow G (2014) Improving beef color stability: practical strategies and underlying mechanisms. Meat Sci 98(3):490-504

11. Hughes JM, Oiseth SK, Purslow PP, Warner RD (2014) A structural approach to understanding the interactions between colour, water-holding capacity and tenderness. Meat Sci 98(3):520-532

12. Yancey JWS, Wharton MD, Apple JK (2011) Cookery method and end-point temperature can affect the warner-bratzler shear force, cooking loss, and internal cooked color of beef longissimus steaks. Meat Sci 88(1):1-7

13. Suman SP, Nair MN, Joseph P, Hunt MC (2016) Factors influencing internal color of cooked meats. Meat Sci 120:133-144

14. Datta AK (2016) Toward computer-aided food engineering: mechanistic frameworks for evolution of product, quality and safety during processing. J Food Eng 176:9-27 
15. Ling B, Tang J, Kong F, Mitcham EJ, Wang S (2014) Kinetics of food quality changes during thermal processing: a review. Food Bioprocess Technol 8(2):343-358

16. Goñi SM, Salvadori VO (2011) Kinetic modelling of colour changes during beef roasting. Proc Food Sci 1:1039-1044

17. Kondjoyan A, Kohler A, Realini CE, Portanguen S, Kowalski R, Clerjon S, Gatellier P, Chevolleau S, Bonny JM, Debrauwer L (2014) Towards models for the prediction of beef meat quality during cooking. Meat Sci 97(3):323-331

18. León K, Mery D, Pedreschi F, León J (2006) Color measurement in lab units from rgb digital images. Food Res Int 39(10):1084-1091

19. Martens H, Stabursvik E, Martens M (1982) Texture and colour changes in meat during cooking related to thermal denaturation of muscle proteins. J Texture Stud 13(3):291-309
20. Pakula C, Stamminger R (2012) Measuring changes in internal meat colour, colour lightness and colour opacity as predictors of cooking time. Meat Sci 90(3):721-727

21. Mancini RA, Hunt MC (2005) Current research in meat color. Meat Sci 71(1):100-121

22. Abril M, Campo MM, Önenç A, Sañudo C, Albertí P, Negueruela AI (2001) Beef colour evolution as a function of ultimate $\mathrm{pH}$. Meat Sci 58(1):69-78

23. Rabeler F, Feyissa AH (2018) Kinetic modeling of texture and color changes during thermal treatment of chicken breast meat. Food Bioprocess Technol 11(8):1495-1504 